\title{
Preface to special issue on progress of computational seismology*
}

\author{
Xiaofei Chen \\ School of Earth and Space Science, University of Science and Technology of China, Hefei 230026, China
}

Seismology had emerged as a discipline of modern science during the last century through an extensive interplay between theoretical and experimental seismological researchers (Aki and Richards, 2002). Today, Seismology is marching into a new era to meet both societal and its intrinsic scientific needs. During such course, both substantial observational study and well-knit theoretical study, and the effective interactions between them are still crucial. Remarkable progress in the aspect of observational seismology have been archived during the past three decades since the revolutionary digital broadband seismometers were deployed around the world, and vast high-quality seismic data have been gained since then and are continuously increasing with an exponential rate. However, how we can exploit new findings of the structure of Earth's interior, or new understanding of earthquake from these tremendously rich seismic data, utterly depends on the study of quantitative seismology. Since almost no exact or analytical solution exist for a more realistic earth model or earthquake model, numerical computation becomes a must for quantitative seismic study. In such sense, theoretical seismic study could not stand along without the significant integrant of numerical computation. Therefore, computational seismology is core of the quantitative seismology.

Due to the extremely rapid development of computing technology, especially the cheaper High Performance Computing platform based on Beowulf PC Cluster technique, numerous progresses have been made in the study of computational seismology during the past decades. Ranging from the studies of seismic hazards mitigation based on seismic strong motion simulations, dynamic rupture modeling of earthquake, finite-frequency tomography, to waveform imaging, form main subjects of the computational seismology. In China, thousands of digital broadband seismometers have been deployed during last decade; accordingly vast of

^Corresponding author. e-mail : xfchen1@ustc.edu.cn

(C) The Seismological Society of China and Springer-Verlag Berlin Heidelberg 2010. high-quality seismic data are acquired. To better interpret those data, promoting the study of computational seismology in China is equally important as the effort made in promoting the seismological observation. This special issue of "Progress of Computational Seismology" is an endeavor for such intention. This special issue contains eight papers and encompasses several different and important theoretical computational seismological problems, including seismic wave modeling using different methods, simulation of the coupled seismic and electromagnetic waves, seismic rupture dynamics and the computing of co-seismic deformation.

Modeling seismic wave-field of global Earth model is important in understanding the physical process of elastic wave propagation through heterogeneous whole Earth. It provides potential forward modeling tools for waveform inversion to study global structure of the Earth's interior. In the article of "Solving singularity in global seismic wavefield simulation using staggered -grid pseudospectral method", Wang and Takenaka present their new progress made in this direction. They proposed an effective hybrid scheme, the staggered-grid pseudospectral method, by elaborately extending their ingenious treatment proposed earlier (Wang et al, 2001) on the singularity arises at the center of the Earth where $r=1$ since the $1 / r$ term appears in the wave equations.

The main issue in modeling of seismic wave propagation at local distance is the strong lateral heterogeneity of Earth model. Therefore, appropriate theory and computational methods are needed. The next four articles in this special issue belong to the efforts in this subject. Zhou et al present a new and more efficient method to calculate the seismic waves propagating through an irregular multi-layered Earth model based on boundary integral equation and the discrete wavenumber algorithm (Bouchon, 2003). Since main calculation concentrated on the localized irregular part of interface, their new method is very efficient in computation while keeping the same accuracy compared with Bouchon- 
Campillo's method.

In a different way, $\mathrm{Yu}$ et al attempts to improve the computational efficiency of boundary element method by applying Born series operator, a neat technique originally proposed by Schuster (1985), instead of directly computing inverse of a full matrix. As showed in the article, this technique indeed significantly improves the computing efficiency, thus makes the boundary element method more applicable in wider range.

Normally, boundary methods is advantageous for models composed of a number of homogeneous regions which are separated by sharp boundaries, e.g., the irregular multi-layered model studied in Zhou et al and Yu et al, therefore its limitation to wide range application is obvious. The article by Ge presents an effort to break such limit. By incorporating an approximate analytical Green's function for the model with a constant-gradient velocity, Ge extends the boundary element method to the model composed of a number of linearly varied heterogeneous regions and shows a particular application for studying the seismic response of sedimentary basins with constant-gradient velocity.

The article by Zhang deals with the scattering of seismic waves by the canyons composed of multiple circular-arc-shaped layers subjected an oblique incident plane $\mathrm{P}$ wave. He extends the Fourier-Bessel wave function series method (Trifunac, 1973) to this particular model and derives analytic solution given in a Fourier-Bessel series. Although the model and solution are too specific and are difficult to apply realistic problems, they can be used to check and calibrate the precision of other numerical methods.

The article of "A new numerical technique for simulating the coupled seismic and electromagnetic waves in layered porous media" by Ren et al in this special issue presents their recent study on seismoelectric effects. Seismoelectric phenomenon has recently attracted much attention among both earthquake seismologists and exploration seismologists. How to effectively solve the coupled equations is still a central issue for this subject. Ren et al successfully extends the generalized reflection-transmission coefficient method for planar multilayered elastic model to the coupled seismoe- lectric problem, and has obtained the solutions for such complicated coupling.

Modeling the rupture dynamics of earthquake fault model is a cutting edge subject of seismology, especially the rupture dynamics of a complex non-planar fault system. Zhang et al present their newest research progress in this filed. Based on Zhang and Chen's early work (2006), they derive the detailed formulas for this problem which are certainly the base for next step computation.

The last article of this special issue is "Controls on the coseismic deformation of the $2008 M_{\mathrm{S}} 8.0$ Wenchuan earthquake in China" by Li and Huang. They investigate the coseismic displacement and deformation of the 2008 Wenchuan earthquake by means of finite element simulation. Except in a narrow strip around the rupture zone, their calculated coseismic displacements are consistent with that from GPS observations and InSAR interpretations. Their numerical study also shows that rupture slip vectors and elastic properties have profound influences on the surface coseismic deformation.

Computational seismology is one of most important subjects of the journal of Earthquake Science, contributions in this aspect to this journal is always more than welcome.

Finally, we thank the following colleagues for reviewing the papers and providing constructive comments: Chaoying Bai, Guohua Gu, Jianwen Liang, Zhongxian Liu, Chengyu Sun, Yanbin Wang, Qingliang Wang, Dinghui Yang, Haiming Zhang, Zhixin Zhao, Xuyao Zheng, Hong Zhou, Zhenghua Zhou.

\section{References}

Aki K and Richards PG (2002). Quantitative Seismology. University Science Books, Sausalito, California.

Bouchon M (2003). A review of the discrete wavenumber method. Pure Appl Geophys 160(3-4): 445-465.

Schuster G T (1985). A hybrid BIE+Born series modeling scheme: Generalized Born series. J Acoust Soc Amer 77: 865-879.

Trifunac M D (1973). Scattering of plane SH waves by a semi-cylindrical canyon. Earthq Eng Struct Dynam 1: 267-281.

Wang Y B, Takenaka H and Furumura T (2001). Modelling seismic wave propagation in a two-dimensional cylindrical whole-earth model using the pseudospectral method. Geophys J Int 145: 689-708.

Zhang H M and Chen X F (2006a). Dynamic rupture on a planar fault in three-dimensional half space. I. Theory. Geophys J Int 164: 633-652. 